
\title{
$\begin{array}{ll}\text { Research Square } & \begin{array}{l}\text { Preprints are preliminary reports that have not undergone peer review. } \\ \text { They should not be considered conclusive, used to inform clinical practice, } \\ \text { or referenced by the media as validated information. }\end{array}\end{array}$
}

\section{Preliminary results using PET/MR in glioblastoma patients treated with regorafenib: an 18F-FET and DWI-ADC comparison}

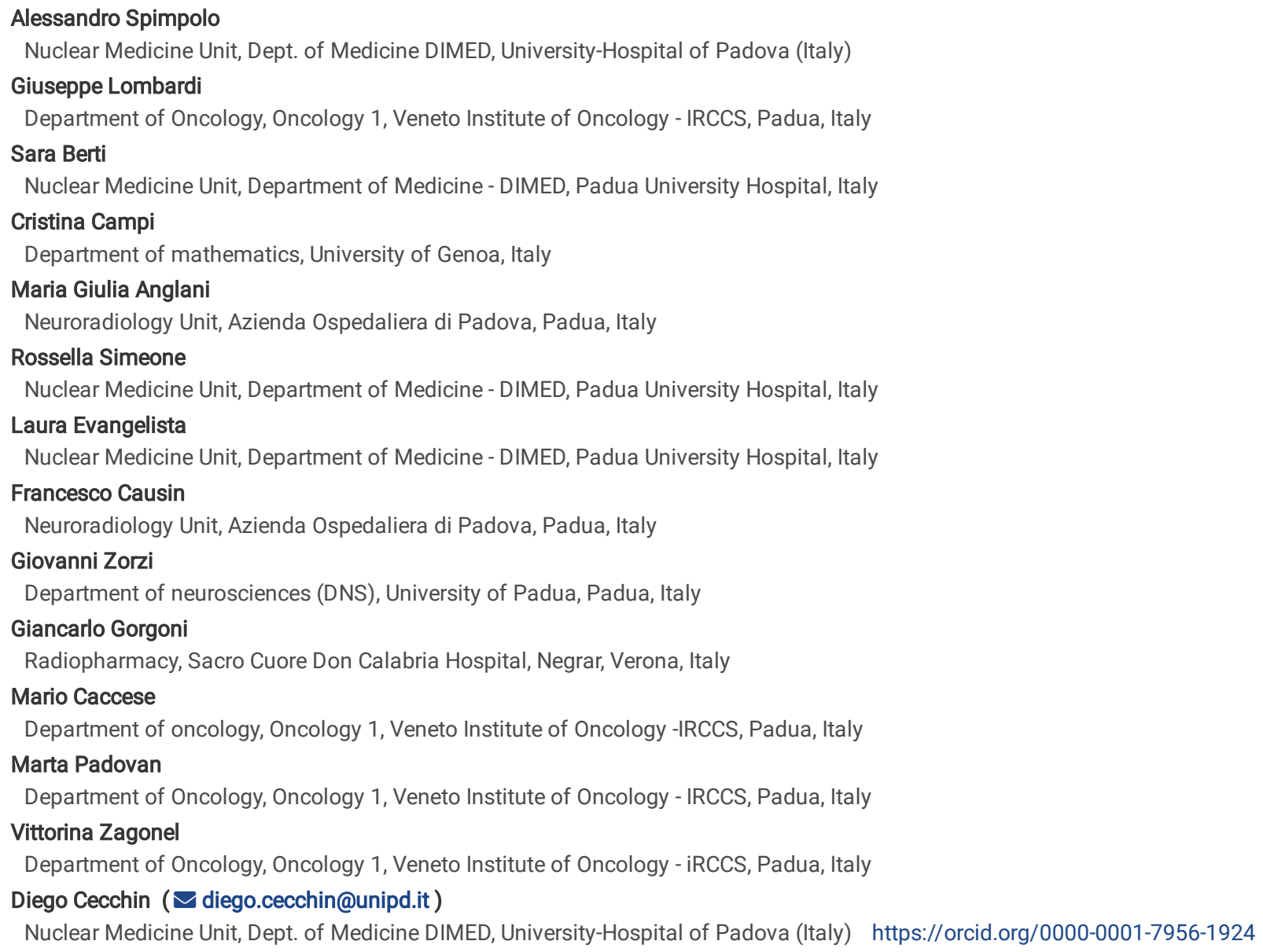




\section{Abstract}

Introduction: The use of regorafenib in recurrent glioblastoma patients has been recently approved by the Italian Medicines Agency (AIFA) and added to the National Comprehensive Cancer Network (NCCN) 2020 guidelines as a preferred regimen. Given its complex effects at the molecular level, the most appropriate imaging tools to assess early response to treatment is still a matter of debate. DWI and ${ }^{18} \mathrm{~F}-\mathrm{FET}$ PET are promising methodologies providing additional information to the currently used RANO criteria. The aim of this study was to evaluate the variations in DWI/ADC- and ${ }^{18} \mathrm{~F}$-FET PET-derived parameters in patients who underwent PET/MR at both baseline and soon after starting regorafenib.

Method: We retrospectively selected 16 consecutive GBM patients who underwent ${ }^{18} \mathrm{~F}-\mathrm{FET}$ PET/MR before and after two cycles of regorafenib. Patients were sorted into stable (SD) or progressive disease (PD) categories in accordance with RANO criteria. We were also able to analyze 4 SD patients who underwent a third PET/MR after another 4 cycles of regorafenib. ${ }^{18} \mathrm{~F}-\mathrm{FET}$ uptake greater than 1.6 times the mean background activity was used to define an area to be superimposed on an $\mathrm{ADC}$ map at baseline and soon after treatment. A number of metrics were then derived and compared.

Result: The average increases in FET and ADC pathological volumes were higher in PD than in SD patients, although in neither case did the difference reach significance. However, when the percentage difference in FET volumes was plotted against the corresponding percentage difference in ADC, a correlation was observed $(R=0.54)$. Patients with a twofold increase in FET after regorafenib showed a significantly higher increase in ADC pathological volume than the remaining subjects $(p=0.0023)$.

Conclusion: In recurrent glioblastoma patients treated with regorafenib, ${ }^{18} \mathrm{~F}-\mathrm{FET}$ and ADC metrics, being obtained from completely different measures, could serve as semi-quantitative independent biomarkers of response to treatment. These promising parameters should be tested in a larger cohort of glioblastoma patients treated with regorafenib.

\section{Introduction}

Glioblastoma multiforme (GBM), the most common primary malignant brain tumor in adults, still carries a dismal prognosis, with a median overall survival of less than 24 months, even after maximal safe resection, concomitant chemoradiotherapy and adjuvant temozolomide [1-3]. In the setting of disease relapse, which is frequent even after aggressive first-line therapies, the use of regorafenib has been recently approved by the Italian Medicines Agency (AIFA) and added to the new National Comprehensive Cancer Network (NCCN) 2020 guidelines as a preferred regimen, based on promising results from a multicenter phase 2 trial (REGOMA) comparing this new drug with a lomustine regimen [4]. Regorafenib is an orally available multi-kinase inhibitor with several molecular targets involved in angiogenesis (VEGFR1-3 and TIE2), oncogenesis (KIT, RET, RAF1, and BRAF) and maintenance of the tumoral microenvironment (PDGFR and FGFR) [5-7]. Given the complexity of its effects at the molecular level, the choice of the most appropriate imaging parameters to be used with patients treated with regorafenib is still a matter of debate. Currently, the recommendations of the Response Assessment in Neuro-Oncology (RANO) Study Group are widely used in both clinical practice and research settings and were also implemented in the REGOMA trial [4,8]. The RANO criteria are based on measurement of areas of contrast-enhancement on post-gadolinium T1-weighted sequences and of non-enhancing disease captured on T2-weighted/FLAIR images. This approach, however, has already been shown to have several limitations and shortcomings in patients treated with anti-angiogenetic drugs, such as bevacizumab, given the normalization of vascular permeability and the related decrease in contrast enhancement induced by these agents [9-11]. In fact, up to $40 \%$ of patients treated with bevacizumab show seemingly stable contrast-enhancing disease with an increase in T2-weighted/FLAIR signal abnormalities, indicating disease progression [12]. Moreover, the lack of a quantifiable measure of non-enhancing disease progression, and the confounding effect of radiation therapy, ischemic injury, and postoperative changes on FLAIR images further complicate the issue.

Diffusion-weighted imaging is a promising methodology that could improve the assessment of treatment response in GBM, thereby extending the existing RANO criteria [13]. It is based on measuring the Brownian motion of water molecules and the various constraints that hamper this physical phenomenon in live tissues. Moreover, DWI-derived apparent diffusion coefficient (ADC) maps offer quantitative information related to tumor cellularity and have already been used in glioma patients to detect the presence of neoplastic tissue in the peritumoral edema [14,15]. Since necrosis, ischemia and inflammation are known to influence water diffusion, heterogeneous ADC values are usually evident in tumoral areas, especially after treatment [16,17]. Consequently, the mean ADC values of one area can fail to depict the spatial heterogeneity of brain tumors, although histogram analysis has already been successfully used as a possible workaround $[17,18]$.

O-(2-18 F-fluoroethyl)-L-tyrosine $\left({ }^{18} \mathrm{~F}-\mathrm{FET}\right)$, an amino acid tracer used in positron emission tomography (PET), is another important tool routinely used for therapy assessment during antiangiogenetic treatment $[19,20]$. Even though several studies have already demonstrated the additional value of amino acid PET over conventional MR-based assessment in this setting [21,22], the interplay between ADC and ${ }^{18}$ F-FET PET in patients treated with regorafenib has not been elucidated so far.

The aim of this study was to evaluate the variations in DWI/ADC- and ${ }^{18} \mathrm{~F}-\mathrm{FET}$ PET-derived parameters in patients undergoing PET/MR both at baseline and soon after beginning regorafenib.

\section{Materials And Methods}

This was a single-center, retrospective, observational study conducted in accordance with the Declaration of Helsinki and after formal approval by our local Ethics Committee (protocol number: AOP1673 - 4831/AO/20). All patients gave written informed consent before undergoing the ${ }^{18} \mathrm{~F}-\mathrm{FET}$ PET/MR, including 
access to their data for research purposes.

2.1 Patient selection

We retrospectively selected sixteen consecutive recurrent GBM patients who underwent ${ }^{18}$ F-FET PET/MR from May 2019 to October 2020 at the Nuclear Medicine Unit of Padua University Hospital before and after two cycles of regorafenib; 4/16 patients were followed up with a third PET/MR; all of the patients were treated at the Veneto Institute of Oncology-IRCCS in Padua. Excluded Patients were those who underwent ${ }^{18}$ F-FET PET/MR but lacked one of the following inclusion criteria:

1. Histologically confirmed glioblastoma;

2. Radiologically and/or histologically confirmed disease relapse after conventional treatment according to RANO criteria (maximal safe resection followed by chemoradiotherapy);

3. Acquisition of baseline ${ }^{18} \mathrm{~F}-\mathrm{FET}$ PET/MR no sooner than one week before starting regorafenib;

4. Acquisition of a second ${ }^{18} \mathrm{~F}-\mathrm{FET}$ PET/MR no later than two weeks after two cycles of regorafenib;

5. No treatment changes between baseline and post-regorafenib ${ }^{18} \mathrm{~F}-\mathrm{FET}$ PET/MR.

\subsection{Image acquisition and reconstruction}

All ${ }^{18}$ F-FET PET/MR images were acquired with a 3-T Biograph integrated PET/MR scanner (Siemens Healthcare, Germany) at the Nuclear Medicine Unit of Padua University Hospital, Italy. Following the most recent recommendations by the European Association of Nuclear Medicine, all study patients were required to fast for a minimum of 4 hours before the intravenous administration of approximately $250 \mathrm{MBq}$ of ${ }^{18} \mathrm{~F}-\mathrm{FET}$. Dynamic PET data were acquired from the time of tracer administration to 50 minutes post-injection [23], while at the same time a standardized MR protocol was performed. The latter included: 1-mm isotropic 3D T1-weighted magnetization-prepared rapid acquisition gradient echo (MPRAGE) (TR 2400 ms, TE 3.24 ms, slice thickness 1 $\mathrm{mm}$, matrix size $256 \times 256$, FOV $256 \times 256 \mathrm{~mm}$ ) before and after contrast enhancement, 3D isovolumetric fluid-attenuated inversion-recovery (FLAIR) (TR 5000 ms, TE 394 ms, TI 1800 ms, slice thickness 1 mm, matrix size 256 x 256, FOV 250 x 250 mm) and RESOLVE® sequence (Siemens Healthcare, Germany) (TR 5,000 ms, TE1 72 ms, TE2 122 ms, voxel size 1.56 x 1.56 x $3.12 \mathrm{~mm}$ ), a high-resolution DWI sequence based on a readout-segmented echoplanar imaging (EPI) strategy [24]. Apparent diffusion coefficient (ADC) images were calculated from acquired DWI images with a b-value of $1000 \mathrm{~s} / \mathrm{mm}^{2}$ and $0 \mathrm{~s} / \mathrm{mm}^{2}$. The contrast medium used with all patients was gadobutrol $0.1 \mathrm{mmol} / \mathrm{Kg}$ (Gadovist ${ }^{\circledR}$, Bayer Inc., Mississauga, Ontario).

A reconstruction of single frame PET images obtained at 20 to 40 minutes after tracer injection was used for the present study as suggested by EANM guidelines. The analysis of the 50 min dynamic curve pattern is beyond the scope of the present paper. Standard corrections for decay, scatter and dead time were performed. A clinical UTE sequence (Siemens Healthcare, Germany) was included in the MR protocol (because more advanced AC methods are limited to a research settings and not directly applicable to a standard clinical setting) and used for attenuation correction of PET. The quality of the derived UTE map was visually assessed in all patients. The PET data were reconstructed using a 3D ordered subset expectation maximization algorithm with 8 iterations, 21 subsets and a 3-mm Gaussian filter, from which PET images with a $256 \times 256$ matrix size (voxel size $=2.32 \times 2.32 \times 2.03 \mathrm{~mm})$ were derived.

\subsection{Qualitative image analysis}

One neuroradiologist and one nuclear medicine physician (with 7 and more than 10 years' experience in the field of neuro-oncology, respectively), blind to the patients clinical outcomes and the follow-up imaging, jointly reviewed all ${ }^{18} \mathrm{~F}-\mathrm{FET} \mathrm{PET} / \mathrm{MR}$ images at both baseline and post-regorafenib. In accordance with the latest Response Assessment Criteria for High-Grade Gliomas by the Response Assessment in Neuro-Oncology (RANO) Working Group [11,25], study patients were divided into the following response-assessment categories: Complete Response (CR), Partial Response (PR), Stable Disease (SD), Progressive Disease (PD).

In cases of assumed CR or PR at the post-regorafenib time point, a follow-up MR scan was performed at least 4 weeks later and reviewed for confirmation. Progressive Disease was defined (according to RANO) as the fulfillment of one or more of the following conditions:

1. $\geq 25 \%$ increase in the sum of the products of the perpendicular diameters of the enhancing lesions compared with the smallest tumor measurement at baseline;

2. Appearance of any new contrast-enhancing lesion;

3. Significant increase in T2/FLAIR non-enhancing lesion.

Patients fell into the Stable Disease category if they did not meet the conditions for complete response, partial response, or progressive disease, and were administered the same or a lower dose of corticosteroids.

\subsection{Image data processing}

The images were imported into PMOD (PMOD® Technologies LLC, Zurich, Switzerland) for VOI delineation.

${ }^{18}$ F-FET PET (FET), post-contrast 3D T1-weighted MPRAGE (MDC) and ADC images were rigidly aligned to the pre-contrast 3D T1-weighted MPRAGE (T1). 
The mean SUV of a crescent-shaped VOI $\left(\mathrm{BG}_{\mathrm{FET}}\right)$, manually drawn in the hemisphere contralateral to the tumor, was used as the ${ }^{18} \mathrm{~F}-\mathrm{FET}$ background [26]. The pathological FET volume ( $\mathrm{FET}_{\text {vol/pat }}$ ) was segmented through a $3 \mathrm{D}$ semiautomatic contouring process, excluding areas with an ${ }^{18} \mathrm{~F}-\mathrm{FET}$ uptake less than 1.6 times the background mean activity. This threshold was based on an ${ }^{18} \mathrm{~F}$-FET biopsy-controlled study, where it was proven to accurately differentiate between tumoral and non-tumoral tissue [27]. The derived segmented volume was visually refined to exclude areas of non-specific ${ }^{18} \mathrm{~F}-\mathrm{FET}$ spillover (major blood vessels, cranial bones, meninges etc.) using the aligned MDC images as the morphological reference.

$\mathrm{FET}_{\text {vol/pat }}$ was then superimposed onto the ADC images (Figure 6) to obtain the corresponding ADC volume (ADC vol). The details are as follows:

1. $\mathrm{FET}_{\text {vol/pat }}$ was imported into the aligned $\mathrm{ADC}$ image;

2. Areas with non-specific high ADC values were automatically subtracted (with the aim also to correct for anatomical distortions induced by metal implants and air filled cavities) from the original volume, pinpointing the ADC values in the cerebrospinal fluid of the lateral ventricles;

3. Areas of the original volume located outside the brain parenchyma were analogously subtracted, pinpointing a region external to the head.

A standard spherical volume (radius $=5 \mathrm{~mm}$ ) was then placed on the ADC images in the hemisphere contralateral to the tumor, carefully avoiding lateral ventricles and major vessels, in order to derive the mean $A D C$ value of the normal brain parenchyma $\left(B G_{A D C}\right)$. This method was chosen in view of the stability of the ADC values in the "healthy" brain parenchyma during treatment with antiangiogenetic agents [13]. A qualitative assessment of the high resolution DWI and ADC derived maps was performed in every patient and revealed no significant distortions or misregistrations affecting the selected tumor area or background area.

The quality of alignment and segmentation was finally checked by an experienced nuclear medicine physician (with more than 10 years' experience in the field of neuro-oncology).

\subsection{Data analysis}

A pixel dump of $F E T_{\text {vol/pat }}, B_{F E T}, A D C_{v o l}$, and $B G_{A D C}$ was imported into the $R$ software [28] for further analyses. The mean $A D C$ value of the $B G_{A D C}$ was used as a threshold for $A D C_{v o l}$. Only those pixels below the threshold were considered pathologic $\left(A D C_{v o l} / p a t\right)$. The percentage differences in $A D C_{v o l} / p a t$ and $\mathrm{FET}_{\text {vol/pat }}$ before and after regorafenib were calculated and compared $\left(\triangle \mathrm{ADC} \mathrm{vol}_{\mathrm{pat}}=\mathrm{ADC}_{\mathrm{vol} / \mathrm{pat}}(\mathrm{T} 1-\mathrm{T} 0) / \mathrm{TO}\right.$ and $\Delta \mathrm{FET}$ vol/pat $=\mathrm{FET}$ vol/pat $\left.(\mathrm{T} 1-\mathrm{T} 0) / \mathrm{T} 0\right)$.

\subsection{Statistical analysis}

All statistical analyses were performed using the R Software. The Shapiro-Wilk normality test was performed on the distribution of all the parameters. Where normal distributions could not be assumed, non-parametric tests were performed. The percentage changes in $\mathrm{ADC}_{\mathrm{vol} / \mathrm{pat}}$ and $\mathrm{FET}$ vol/pat ${ }_{\text {before and after }}$ regorafenib were plotted and the Pearson correlation coefficient (PCC) calculated, assuming a linear correlation between the two variables. The differences in the percentage changes in $\mathrm{FET}_{\mathrm{vol} / \mathrm{pat}}$ and $\mathrm{ADC}_{\mathrm{vol} / \mathrm{pat}}$ between the response groups determined according to RANO criteria [11] were compared using the Wilcoxon signed-rank test for repeated measures. The significance level (a) was set at 0.05 .

\section{Results}

\subsection{Patients}

Our study population consisted of 15 IDH-wt and 1 glioblastoma NOS patients ( 6 females, 10 males, median age: 54.4 years, age range: 31 - 73 years). All the study patients had undergone maximal safe resection, adjuvant chemoradiotherapy with temozolomide and subsequent maintenance temozolomide (from 1 to 12 cycles) before relapsing. The median time elapsed between radiotherapy and baseline ${ }^{18}$ F-FET PET/MR was 319 days. In one subject, reirradiation was given in a single fraction about 4 weeks before starting regorafenib. Eight of the 16 patients had been surgically retreated before being scheduled for regorafenib, and at least 19 days passed before the first ${ }^{18} \mathrm{~F}-\mathrm{FET}$ PET/MR was performed. All the study patients received 2 cycles of regorafenib (160 mg per day; 3 weeks on, 1 week off) without treatment interruption. The characteristics of the population are summarized in Table 1.

\section{$3.2^{18} \mathrm{~F}-\mathrm{FET}$ PET/MR image analysis}

After 2 cycles of regorafenib, $7 / 16$ (44\%) patients were observed to have stable disease (SD), and the remaining 9/16 (56\%) to have disease progression (PD) according to the RANO criteria (Table 2). The values of the ${ }^{18} \mathrm{~F}-\mathrm{FET}$ PET/MR-derived parameters before and after treatment with regorafenib (FET ${ }_{\text {vol/pat }}$ $\mathrm{TBR}_{\text {mean }}, \mathrm{TBR}_{\max }$ [23], $\mathrm{ADC}_{\mathrm{vol} / \text { pat }}$, and mean $\mathrm{ADC}_{\mathrm{vol} / \mathrm{pat}}$ ) are listed in Table 2 and summarized in Table 3. Their absolute and percentage variations after treatment are presented in Table 4. Although the absolute and percentage increases in $\mathrm{FET}_{\text {vol/pat }}$ were on average higher in PD than SD patients (21605 $\mathrm{mm}^{3}$ and $168 \%$ versus $-1160 \mathrm{~mm}^{3}$ and $70 \%$ ), the differences between the two groups were not statistically significant ( $\left.\mathrm{p}=0.17\right)$. Similarly, the average absolute and percentage increases in ADC pathological volume were also higher in PD than SD subjects ( $501 \mathrm{~mm}^{3}$ and $554 \%$ versus $33 \mathrm{~mm}{ }^{3}$ and $297 \%$ ), and also failed to reach statistical significance $(p=0.53)$. The percentage variations in mean $A D C, T_{B R}$ max and TBR mean did not differ significantly between SD and PD patients (Tables 3 and 4, Figure 1). When the percentage difference in FET pathological volumes was plotted against the corresponding percentage difference in ADC pathological volumes, a linear regression model revealed a correlation between the two variables $(R=0.54)($ Figure 2$)$. We found no evident correlation between the percentage variation in mean ADC values and the corresponding percentage variation in $F E T_{\text {vol }} /$ pat $(R=0.04)$. Patients with at least a twofold increase in FET pathological volume (Figure 3) after regorafenib showed a significantly higher increase in ADC pathological volume than the remaining subjects $(p=0.0023)$. In $2 / 9$ subjects classified as progressive after two cycles of regorafenib, the FET pathological volume 
decreased by $76 \%$ and $31 \%$, respectively. Consistent with this, a decrease in the ADC pathological volume was observed in the former (-93\%), while no residual pathological ADC areas could be detected in the latter. In contrast, in 3/7 patients classified as stable after treatment, an increase in FET pathological volume $\left(5895 \mathrm{~mm}^{3}, 2057 \mathrm{~mm}^{3}\right.$, and $1009 \mathrm{~mm}^{3}$, respectively) was observed; in the same patients, the ADC pathological volume increased at similar rates $\left(273 \mathrm{~mm}^{3}, 88 \mathrm{~mm}^{3}\right.$, and $\left.425 \mathrm{~mm}^{3}\right)$.

\section{Discussion}

The main finding to emerge from the present study was the correlation between the percentage changes in the pathological FET and ADC volumes in recurrent GBM patients treated with regorafenib at their first disease relapse. To our knowledge, this is the first work assessing the variation in the $A D C$ signal in the FET-positive volume in patients undergoing ${ }^{18} \mathrm{~F}$-FET PET/MR both at baseline and soon after beginning this new beneficial second-line therapy. The value of DWI-derived parameters in treatment monitoring of GBM patients has already been extensively investigated [29-32], and many authors have suggested that the DWI methodology could play an important role in guiding response assessment, particularly when conventional contrast-enhanced and T2-weighted/FLAIR sequences are less reliable. Although DWI sequences are routinely acquired as part of the standard MR protocol for brain tumor imaging, the most recent recommendations [33] only describe how diffusion-weighted images should be acquired and provide no guidance for clinically interpreting and quantifying the extent of the tumor for the purpose of response evaluation. Two major issues consequently arise, the first regarding the strategy to identify the region on the DWI-ADC images to be analyzed, the second regarding the threshold for pathological ADC values. In most of the published studies, the tumor volume was outlined and the VOI constructed on contrast-enhanced T1-weighted images, which were subsequently transferred to the corresponding DWI-ADC images. Buemi et al. [34], for example, manually drew the VOls encompassing the areas of tumor-related contrast enhancement, and T2-weighted/FLAIR abnormalities were mapped onto the corresponding ADC images, thus deriving the CE-ADC and T2/FLAIR-ADC volumes, respectively. Histogram analysis and curve fitting using a two-mixture normal distribution model were carried out to calculate the mean $A D C$ of the lowest $A D C$ values in these areas (CE-ADC-L and T2/FLAIR-ADC-L) [35]. Interestingly, only the mean ADC in CE-ADC-L turned out to be significantly predictive of progression-free survival and overall survival in GBM patients treated with bevacizumab and fotemustine. The predictive value of the low-ADC areas is confirmed by other published papers [36,37]. Zeiner et al. [38] calculated the ADC-ratio by measuring the minimum ADC values in the tumor and normalizing them by the ADC values of the contralateral, normal appearing brain tissue. In our study, instead, a standard VOI contralateral to the lesion was used to determine the appropriate threshold for the selection of pathological ADC values. This approach allowed for a more direct identification of the low ADC values in the defined VOIs, avoiding the need for complex mathematical models. However, the post-regorafenib variation in the mean ADC thus calculated did not significantly correlate with the corresponding change in FET-positive volume, nor with the RANO response categories. A possible explanation for this discrepancy may lie in the different methodological approaches used here and in the previously published studies [13,34,43-45,35-42]. This highlights the importance of future efforts towards standardizing the analysis of ADC maps before considering the inclusion of this methodology in the response assessment criteria.

It is important to note that the changes in neither the pathological FET volume nor the pathological ADC volume were significantly different in the stable and progressive patients as assessed by RANO criteria. These criteria are based mostly on changes in T2/FLAIR and contrast-enhanced areas, which are known to be affected by edema, inflammation, gliosis, and disruption of the blood-brain barrier. DWI, instead, is sensitive to microscopic water motion, resulting in relatively restricted diffusion in areas of tightly packed tumor cells. However, diffusion may be altered by causes other than increased cellularity in neoplastic tissue, and the diagnostic performance of this methodology is influenced by the choice of the appropriate DWI parameter to analyze [17,46,47]. Moreover, the heterogeneity of the ADC signal may have translated into the wide variability we observed in the changes in the pathological $A D C$ volume after regorafenib. This, in turn, may explain why a relatively high threshold of increase in FET pathological volume was needed to subdivide our population into groups with significantly different pathological ADC volumes.

In three cases which were classified according to the RANO criteria as stable after treatment with regorafenib, both the ADC and FET pathological volumes increased compared with the baseline examinations (patients \#5, \#8, and \#13; Figure 5). Information from subsequent follow-ups was available for two of these patients: a) patient \#5 (interestingly, classified as SD by the RANO criteria) showed a slight increase in $\mathrm{FET}_{\text {vol/pat }}$ (and to a lesser extent also in $\mathrm{ADC}_{\mathrm{vol} / \mathrm{pat}}$ ) at a PET/MR examination performed two months later (TP3 in Fig 4), and presented disease progression at an MR scan performed 4 months later; b) patient \#8 showed a significant increase in $\mathrm{FET}_{\text {vol/pat }}$ (and to a lesser extent also in $\mathrm{ADC}_{\mathrm{vol} / \mathrm{pat}}$ ) at a subsequent PET/MR examination (TP3 in Fig 4), and was consistently considered progressive according to the RANO criteria. We were able to carry out a follow-up PET/MR in another two cases (patients \#9 and \#16, both SD at the PET/MR examination after two cycles of regorafenib): a) patient \# 9 presented minimal variations in $\mathrm{FET}_{\mathrm{vol} / \mathrm{pat}}$ and $\mathrm{ADC}_{\mathrm{vol} / \mathrm{pat}}$ after two cycles of regorafenib, and remained stable (presenting a decrease in $\mathrm{FET}_{\mathrm{vol} / \mathrm{pat}}$ and $\mathrm{ADC}_{\mathrm{vol} / \mathrm{pat}}$ ) at the follow-up PET/MR (TP3 in Fig 4); b) patient \#16 showed a significant increase in both $\mathrm{FET}_{\mathrm{vol} / \mathrm{pat}}$ and $\mathrm{ADC}_{\mathrm{vol} / \mathrm{pat}}$ between the PET/MR performed after two cycles of regorafenib (Fig 4) and the follow-up $\mathrm{PET} / \mathrm{MR}$, and was considered progressive according to the RANO criteria.

These four cases, although insufficient to draw definitive conclusions, seem to show consistent variations in FET and ADC pathological volumes in followup examinations performed after six cycles of regorafenib, and confirm the greater predictive value of these parameters compared with the standard RANO criteria. In fact, the variations in FET and ADC predicted the follow-up in three out of four cases.

Moreover, it is notable that an increase in both ADC and FET pathological volumes (in the PET/MR after two cycles of regorafenib) predicted disease progression in subjects \#5 and \#8 (who were categorized as stable according to the RANO criteria). The so identified ${ }^{18} \mathrm{~F}-\mathrm{FET}$ and $\mathrm{ADC}$ areas and values, which are correlated but were obtained from completely different measures, could serve as independent biomarkers of treatment response. It is worth bearing in mind, however, that the RANO criteria include cutoffs for the percentage change in contrast-enhancing tumor volume, and small increases in 
contrast-enhanced volumes are not sufficient to classify a patient as progressive. Therefore, appropriate thresholds should be established for the newly proposed DWI- and FET-derived parameters in prospective case series with adequate outcome measures.

Our study has some limitations, including that regorafenib was introduced only recently:

1. It was retrospective in nature and included only a relatively small number of patients;

2. Adequate follow up was not available for all patients due to the novelty of the treatment;

3. The current RANO criteria were assumed as gold standard.

Despite these limitations, we focused on a highly homogeneous patient population comprising GBM subjects at their first disease relapse, and all patients were treated with a recently approved chemotherapeutic agent (regorafenib). Moreover, all imaging studies were acquired at the same institution with an integrated PET/MR system and a standardized protocol.

\section{Conclusions}

In the present study, we have proposed a method to identify the pathological ADC volume based on the corresponding ${ }^{18} \mathrm{~F}-\mathrm{FET}$ positive region in intrinsically co-registered ${ }^{18}$ F-FET PET/MR images. We found a correlation between the percentage changes in pathological FET and DWI-ADC volumes in glioblastoma patients treated with regorafenib at their first disease relapse. In 4/16 cases followed up with a third PET/MR, the results seemed encouraging compared to the RANO criteria. The ${ }^{18}$ F-FET and ADC metrics identified could, given they were correlated but obtained from completely different measures, serve as semiquantitative independent biomarkers of response to regorafenib treatment.

\section{Declarations}

Compliance with Ethical Standards:

Funding: This research received no external funding.

Disclosure of potential conflicts of interest: All the authors declare no conflict of interest.

Research involving human participants and/or animals: All procedures performed in studies involving human participants were in accordance with the ethical standards of the institutional and/or national research committee and with the 1964 Helsinki declaration and its later amendments or comparable ethical standards. This was a single-center, retrospective, observational study conducted after formal approval by our local Ethics Committee (protocol number: AOP1673-4831/AO/20).

Informed consent: All patients gave written informed consent before undergoing the 18F-FET PET/MR, including access to their data for research purposes.

Data availability: Due to the retrospective nature of this research, participants of this study did not explicitly agree for their data to be shared publicly, so supporting data is not available.

Author Contributions: Conceptualization, A.S., G.L., S.B. and D.C.; methodology, A.S., S.B., C.C., M.G.A. and D.C.; software, S.B. and C.C.; formal analysis, C.C.; investigation, G.L., M.G.A., R.S., L.E., G.G., M.C. and M.P.; data curation, A.S., S.B., C.C. and D.C.; writing-original draft preparation, A.S., S.B. and D.C.; writing -review and editing, G.L., M.G.A., L.E., F.C., G.Z. and V.Z.; visualization, D.C., M.G.A.; supervision, G.L., V.Z. and D.C.; project administration, D.C. All authors read and agreed to the published version of the manuscript.

\section{References}

1. Rønning PA, Helseth E, Meling TR, et al. A population-based study on the effect of temozolomide in the treatment of glioblastoma multiforme. Neuro Oncol 2012;14:1178-1184.

2. Kruchko C, Ostrom QT, Gittleman H, et al. The CBTRUS story: Providing accurate population-based statistics on brain and other central nervous system tumors for everyone. Neuro Oncol 2018;20:295-298.

3. Stupp R, Mason W, Bent M van den, et al. EORTC 22981: Radiotherapy plus concomitant and adjuvant temozolomide for globlastoma. N Engl J Med 2005.

4. Lombardi G, De Salvo GL, Eoli M, et al. REGOMA: A randomized, multicenter, controlled open-label phase II clinical trial evaluating regorafenib (REG) activity in relapsed glioblastoma (GBM) patients (PTS). J Clin Oncol 2017;35:TPS2085-TPS2085.

5. Abou-Elkacem L, Arns S, Brix G, et al. Regorafenib inhibits growth, angiogenesis, and metastasis in a highly aggressive, orthotopic colon cancer model. Mol Cancer Ther 2013;12:1322-1331.

6. Wilhelm SM, Dumas J, Adnane L, et al. Regorafenib (BAY 73-4506): A new oral multikinase inhibitor of angiogenic, stromal and oncogenic receptor tyrosine kinases with potent preclinical antitumor activity. Int J Cancer 2011;129:245-255.

7. Wilhelm SM, Carter C, Tang LY, et al. BAY 43-9006 exhibits broad spectrum oral antitumor activity and targets the RAF/MEK/ERK pathway and receptor tyrosine kinases involved in tumor progression and angiogenesis. Cancer Res 2004;64:7099-7109. 
8. Wen PY, Macdonald DR, Reardon DA, et al. Updated Response Assessment Criteria for High-Grade Gliomas: Response Assessment in Neuro-Oncology Working Group. J Clin Oncol 2010;28:1963-1972.

9. Ellingson BM, Malkin MG, Rand SD, et al. Validation of functional diffusion maps (fDMs) as a biomarker for human glioma cellularity. J Magn Reson Imaging 2010;31:538-548.

10. Wen PY, Cloughesy TF, Ellingson BM, et al. Report of the Jumpstarting Brain Tumor Drug Development Coalition and FDA clinical trials neuroimaging endpoint workshop (January 30, 2014, Bethesda MD). Neuro Oncol 2014;16:vii36-vii47.

11. Wen PY, Macdonald DR, Reardon DA, et al. Updated response assessment criteria for high-grade gliomas: Response assessment in neuro-oncology working group. J Clin Oncol 2010;28:1963-1972.

12. Delgado-López PD, Riñones-Mena E, Corrales-García EM. Treatment-related changes in glioblastoma: a review on the controversies in response assessment criteria and the concepts of true progression, pseudoprogression, pseudoresponse and radionecrosis. Clin Transl Oncol 2018;20:939-953.

13. Auer TA, Breit H-C, Marini F, et al. Evaluation of the apparent diffusion coefficient in patients with recurrent glioblastoma under treatment with bevacizumab with radiographic pseudoresponse. J Neuroradiol = J Neuroradiol 2019;46:36-43.

14. Provenzale JM, McGraw P, Mhatre P, et al. Peritumoral brain regions in glionias and meningiomas: Investigation with isotropic diffusion-weighted MR imaging and diffusion-tensor MR imaging. Radiology 2004;232:451-460.

15. Yamasaki F, Kurisu K, Satoh K, et al. Apparent diffusion coefficient of human brain tumors at MR imaging. Radiology 2005;235:985-991.

16. Smith JS, Cha S, Mayo MC, et al. Serial diffusion-weighted magnetic resonance imaging in cases of glioma: distinguishing tumor recurrence from postresection injury. J Neurosurg 2005;103:428-438.

17. Huang RY, Neagu MR, Reardon DA, et al. Pitfalls in the neuroimaging of glioblastoma in the era of antiangiogenic and immuno/targeted therapy detecting illusive disease, defining response. Front Neurol 2015;6.

18. Kondo M, Uchiyama Y. Apparent diffusion coefficient histogram analysis for prediction of prognosis in glioblastoma. J Neuroradiol 2018;45:236-241.

19. Albert NL, Weller M, Suchorska B, et al. Response Assessment in Neuro-Oncology working group and European Association for Neuro-Oncology recommendations for the clinical use of PET imaging in gliomas. Neuro Oncol 2016;18:1199-1208.

20. Stegmayr C, Oliveira D, Niemietz N, et al. Influence of bevacizumab on blood-brain barrier permeability and O-(2-18F-Fluoroethyl)-L-tyrosine uptake in rat gliomas. J Nucl Med 2017;58:700-705.

21. Hutterer M, Nowosielski M, Putzer D, et al. O-(2-18F-fluoroethyl)-L-tyrosine PET predicts failure of antiangiogenic treatment in patients with recurrent high-grade glioma. J Nucl Med 2011;52:856-864.

22. Galldiks N, DunkI V, Ceccon G, et al. Early treatment response evaluation using FET PET compared to MRI in glioblastoma patients at first progression treated with bevacizumab plus lomustine. Eur J Nucl Med Mol Imaging 2018;45:2377-2386.

23. Law I, Albert NL, Arbizu J, et al. Joint EANM/EANO/RANO practice guidelines/SNMMI procedure standards for imaging of gliomas using PET with radiolabelled amino acids and [ 18 F]FDG: version 1.0. Eur J Nucl Med Mol Imaging 2019;46:540-557.

24. Cohen-Adad J. High-Resolution DWI in Brain and Spinal Cord with syngo RESOLVE. Siemens Magnetom - Clin Neurol 2012;510:16-23.

25. Chukwueke UN, Wen PY. Use of the Response Assessment in Neuro-Oncology (RANO) criteria in clinical trials and clinical practice. CNS Oncol 2019;8:CNS28.

26. Unterrainer M, Vettermann F, Brendel M, et al. Towards standardization of 18F-FET PET imaging: do we need a consistent method of background activity assessment? EJNMMI Res 2017;7:48.

27. Pauleit D, Floeth F, Hamacher K, et al. O-(2-[18F]fluoroethyl)-L-tyrosine PET combined with MRI improves the diagnostic assessment of cerebral gliomas. Brain 2005;128:678-687.

28. R Core Team (2017). R: A language and environment for statistical computing. R Foundation for Statistical Computing, Vienna, Austria.

29. Ellingson BM, Malkin MG, Rand SD, et al. Validation of functional diffusion maps (fDMs) as a biomarker for human glioma J Magn Reson Imaging 2010;31:538-548.

30. Sugahara T, Korogi Y, Kochi M, et al. Usefulness of diffusion-weighted MRI with echo-planar technique in the evaluation of cellularity in gliomas. $J$ Magn Reson Imaging 1999;9:53-60.

31. Chenevert TL. Diffusion Magnetic Resonance Imaging: an Early Surrogate Marker of Therapeutic Efficacy in Brain Tumors. J Natl Cancer Inst 2000;92:2029-2036.

32. Padhani AR, Liu G, Mu-Koh D, et al. Diffusion-weighted magnetic resonance imaging as a cancer biomarker: Consensus and recommendations. Neoplasia, vol. 11, 2009:102-125.

33. Ellingson BM, Bendszus M, Boxerman J, et al. Consensus recommendations for a standardized Brain Tumor Imaging Protocol in clinical trials. Neuro Oncol 2015;17:1188-1198.

34. Buemi F, Guzzardi G, Del Sette B, et al. Apparent diffusion coefficient and tumor volume measurements help stratify progression-free survival of bevacizumab-treated patients with recurrent glioblastoma multiforme. Neuroradiol J 2019;32:241-249.

35. Pope WB, Kim HJ, Alger J, et al. Recurrent Glioblastoma Multiforme: ADC Histogram Analysis Predicts Response to Bevacizumab Purpose: Methods: Results: Conclusion: 2009;252.

36. Pope WB, Lai A, Mehta R, et al. Apparent diffusion coefficient histogram analysis stratifies progression-free survival in newly diagnosed bevacizumabtreated glioblastoma. Am J Neuroradiol 2011;32:882-889. 
37. Pope WB, Qiao XJ, Kim HJ, et al. Apparent diffusion coefficient histogram analysis stratifies progression-free and overall survival in patients with recurrent GBM treated with bevacizumab: A multi-center study. J Neurooncol 2012;108:491-498.

38. Zeiner, Kinzig, Divé, et al. Regorafenib CSF Penetration, Efficacy, and MRI Patterns in Recurrent Malignant Glioma Patients. J Clin Med $2019 ; 8: 2031$.

39. Asao C, Korogi Y, Kitajima M, et al. Diffusion-weighted imaging of radiation-induced brain injury for differentiation from tumor recurrence. AJNR Am J Neuroradiol 2005;26:1455-1460.

40. Chang PD, Chow DS, Yang PH, et al. Predicting Glioblastoma Recurrence by Early Changes in the Apparent Diffusion Coefficient Value and Signal Intensity on FLAIR Images. Am J Roentgenol 2017;208:57-65.

41. Jain R, Scarpace LM, Ellika S, et al. Imaging response criteria for recurrent gliomas treated with bevacizumab: Role of diffusion weighted imaging as an imaging biomarker. J Neurooncol 2010;96:423-431.

42. Kondo M, Uchiyama Y. Apparent diffusion coefficient histogram analysis for prediction of prognosis in glioblastoma. J Neuroradiol 2018;45:236-241.

43. Kim BS, Kim ST, Kim JH, et al. Apparent Diffusion Coefficient as a Predictive Biomarker for Survival in Patients with Treatment-Naive Glioblastoma Using Quantitative Multiparametric Magnetic Resonance Profiling. World Neurosurg 2019;122:e812-e820.

44. La Violette PS, Mickevicius NJ, Cochran EJ, et al. Precise ex vivo histological validation of heightened cellularity and diffusion-restricted necrosis in regions of dark apparent diffusion coefficient in 7 cases of high-grade glioma. Neuro Oncol 2014;16:1599-1606.

45. Mong S, Ellingson BM, Nghiemphu PL, et al. Persistent diffusion-restricted lesions in bevacizumab-treated malignant gliomas are associated with improved survival compared with matched controls. Am J Neuroradiol 2012;33:1763-1770.

46. Langen K-J, Galldiks N, Hattingen E, et al. Advances in neuro-oncology imaging. Nat Rev Neurol 2017;13:279-289.

47. Sundgren PC, Fan X, Weybright $\mathrm{P}$, et al. Differentiation of recurrent brain tumor versus radiation injury using diffusion tensor imaging in patients with new contrast-enhancing lesions. Magn Reson Imaging 2006;24:1131-1142.

\section{Tables}

Table 1: Characteristics of the study population. The 15th subject underwent re-irradiation (20Gy, 1fr, EBRT) before starting regorafenib. GBM = glioblastoma; TMZ = temozolamide; $\mathrm{RTCHT}=$ concomitant radio-chemotherapy; $w t$ = wildtype. 


\begin{tabular}{|c|c|c|c|c|c|c|c|c|c|}
\hline Patient & Sex & Age & Histology & $\begin{array}{l}1^{\text {st }} \\
\text { Treatment }\end{array}$ & $\begin{array}{l}\text { Maintenance } \\
\text { TMZ (cycles) }\end{array}$ & $\begin{array}{l}2^{\text {nd }} \text { Treatment } \\
\text { before regorafenib }\end{array}$ & $\begin{array}{l}\text { Latest Surgery to } 1^{\text {st }} \\
\text { PET/MR (days) }\end{array}$ & $\begin{array}{l}\text { Latest RT } \\
\text { to } \\
1^{\text {st }} \\
\text { PET/MR } \\
\text { (days) }\end{array}$ & $\begin{array}{l}1^{\text {st }} \text { to } 2^{\text {nd }} \\
\text { PET/MR (days) }\end{array}$ \\
\hline 1 & M & 40 & $\begin{array}{l}\text { GBM } \\
\text { IDHwt }\end{array}$ & $\begin{array}{l}\text { Surgery + } \\
\text { RTCHT }\end{array}$ & 6 & Surgery & 42 & 329 & 56 \\
\hline 2 & $\mathrm{~F}$ & 45 & $\begin{array}{l}\text { GBM } \\
\text { IDHwt }\end{array}$ & $\begin{array}{l}\text { Surgery + } \\
\text { RTCHT }\end{array}$ & 12 & & 469 & 405 & 77 \\
\hline 3 & $\mathrm{~F}$ & 58 & $\begin{array}{l}\text { GBM } \\
\text { IDHwt }\end{array}$ & $\begin{array}{l}\text { Surgery + } \\
\text { RTCHT }\end{array}$ & 1 & Surgery & 62 & 1162 & 49 \\
\hline 4 & M & 66 & $\begin{array}{l}\text { GBM } \\
\text { IDHwt }\end{array}$ & $\begin{array}{l}\text { Surgery + } \\
\text { RTCHT }\end{array}$ & 12 & & 504 & 414 & 70 \\
\hline 5 & M & 53 & $\begin{array}{l}\text { GBM } \\
\text { IDHwt }\end{array}$ & $\begin{array}{l}\text { Surgery + } \\
\text { RTCHT }\end{array}$ & 6 & & 284 & 180 & 63 \\
\hline 6 & $M$ & 65 & $\begin{array}{l}\text { GBM } \\
\text { IDHwt }\end{array}$ & $\begin{array}{l}\text { Surgery + } \\
\text { RTCHT }\end{array}$ & 10 & & 406 & 322 & 62 \\
\hline 7 & $\mathrm{~F}$ & 31 & $\begin{array}{l}\text { GBM } \\
\text { IDHwt }\end{array}$ & $\begin{array}{l}\text { Surgery + } \\
\text { RTCHT }\end{array}$ & 3 & & 201 & 123 & 84 \\
\hline 8 & M & 48 & $\begin{array}{l}\text { GBM } \\
\text { IDHwt }\end{array}$ & $\begin{array}{l}\text { Surgery + } \\
\text { RTCHT }\end{array}$ & 6 & Surgery & 41 & 858 & 70 \\
\hline 9 & $\mathrm{~F}$ & 39 & $\begin{array}{l}\text { GBM } \\
\text { IDHwt }\end{array}$ & $\begin{array}{l}\text { Surgery + } \\
\text { RTCHT }\end{array}$ & 6 & & 363 & 312 & 56 \\
\hline 10 & $\mathrm{~F}$ & 48 & $\begin{array}{l}\text { GBM } \\
\text { IDHwt }\end{array}$ & $\begin{array}{l}\text { Surgery + } \\
\text { RTCHT }\end{array}$ & 8 & Surgery & 42 & 314 & 56 \\
\hline 11 & $M$ & 72 & $\begin{array}{l}\text { GBM } \\
\text { IDHwt }\end{array}$ & $\begin{array}{l}\text { Surgery + } \\
\text { RTCHT }\end{array}$ & 6 & Surgery & 55 & 333 & 56 \\
\hline 12 & $M$ & 60 & $\begin{array}{l}\text { GBM } \\
\text { IDHwt }\end{array}$ & $\begin{array}{l}\text { Surgery + } \\
\text { RTCHT }\end{array}$ & 2 & Surgery & 46 & 168 & 64 \\
\hline 13 & $\mathrm{~F}$ & 64 & $\begin{array}{l}\text { GBM } \\
\text { IDHwt }\end{array}$ & $\begin{array}{l}\text { Surgery + } \\
\text { RTCHT }\end{array}$ & 2 & & 406 & 319 & 49 \\
\hline 14 & M & 61 & $\begin{array}{l}\text { GBM } \\
\text { IDHwt }\end{array}$ & $\begin{array}{l}\text { Surgery + } \\
\text { RTCHT }\end{array}$ & 2 & Surgery & 19 & 183 & 56 \\
\hline 15 & $M$ & 48 & $\begin{array}{l}\text { GBM } \\
\text { NOS }\end{array}$ & $\begin{array}{l}\text { Surgery + } \\
\text { RTCHT }\end{array}$ & 12 & Re-irradiation & 3052 & 32 & 84 \\
\hline 16 & M & 73 & $\begin{array}{l}\text { GBM } \\
\text { IDHwt }\end{array}$ & $\begin{array}{l}\text { Surgery + } \\
\text { RTCHT }\end{array}$ & 5 & Surgery & 36 & 239 & 48 \\
\hline
\end{tabular}

Table 2: ${ }^{18} \mathrm{~F}-\mathrm{FET}$ PET/MR parameters at baseline and after 2 cycles of regorafenib. $\mathrm{FET}_{\mathrm{vol} / \mathrm{pat}}$ and $\mathrm{ADC} \mathrm{vol}_{\mathrm{p} \text { pat }}$ are the pathological segmented $\mathrm{FET}$ and $\mathrm{ADC}$

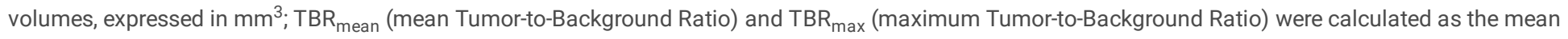
and maximum uptakes, respectively, of the FET-positive area normalized for the mean background uptake. In two of the study subjects at baseline and in one post-regorafenib no pixels remained after ADC normalization. The RANO criteria were used to sort the patients into response categories: SD = Stable Disease; $P D=$ Progressive Disease. 


\begin{tabular}{|c|c|c|c|c|c|c|c|c|c|c|c|}
\hline \multirow[t]{2}{*}{ PT } & \multicolumn{5}{|c|}{ BASELINE ${ }^{18} \mathrm{~F}$-FET PET/MR } & \multicolumn{5}{|c|}{ POST-REGORAFENIB ${ }^{18}$ F-FET PET/MR } & \multirow[t]{2}{*}{ RANO } \\
\hline & FETvol/pat & TBRmean & TBRmax & ADCvol/pat & $\begin{array}{l}\text { mean } \\
\text { ADCvol/pat }\end{array}$ & FETvol/pat & TBRmean & TBRmax & ADCvol/pat & $\begin{array}{l}\text { mean } \\
\text { ADCvol/pat }\end{array}$ & \\
\hline 1 & 4617 & 1.97 & 3.12 & 15 & 586.3 & 10370 & 1.99 & 3.28 & 384 & 604.7 & PD \\
\hline 2 & 17322 & 2.07 & 3.50 & 913 & 563.0 & 13009 & 2.18 & 4.14 & 69 & 579.0 & SD \\
\hline 3 & 3699 & 1.69 & 2.18 & 627 & 666.7 & 8578 & 1.78 & 2.55 & 1280 & 689.7 & PD \\
\hline 4 & 9785 & 1.88 & 3.09 & 1252 & 645.5 & 16413 & 2.07 & 3.43 & 178 & 733.9 & PD \\
\hline 5 & 1242 & 1.83 & 2.33 & 17 & 668.1 & 7137 & 2.11 & 3.67 & 290 & 687.1 & SD \\
\hline 6 & 10710 & 1.79 & 3.42 & 631 & 657.9 & 2548 & 1.69 & 2.11 & 41 & 637.8 & PD \\
\hline 7 & 1873 & 1.79 & 2.35 & 253 & 667.7 & 1486 & 1.82 & 2.39 & 335 & 646.3 & SD \\
\hline 8 & 9052 & 1.79 & 2.47 & 240 & 383.2 & 11109 & 1.79 & 2.77 & 328 & 566.1 & SD \\
\hline 9 & 6173 & 1.99 & 3.50 & 184 & 626.1 & 4007 & 1.87 & 2.77 & 359 & 558.4 & SD \\
\hline 10 & 3941 & 1.87 & 3.25 & 266 & 606.7 & 8475 & 1.94 & 3.20 & 833 & 644.3 & PD \\
\hline 11 & 22115 & 1.98 & 3.44 & / & / & 60399 & 2.19 & 3.40 & 2579 & 640.1 & PD \\
\hline 12 & 39114 & 2.04 & 4.13 & 666 & 402.1 & 111268 & 2.14 & 4.37 & 1571 & 461.5 & PD \\
\hline 13 & 3955 & 1.87 & 2.88 & 398 & 677.3 & 4964 & 1.89 & 2.78 & 823 & 675.4 & SD \\
\hline 14 & 57593 & 2.20 & 4.29 & / & / & 39486 & 2.00 & 3.11 & / & / & PD \\
\hline 15 & 10754 & 1.94 & 3.64 & 234 & 606.2 & 99238 & 2.35 & 4.64 & 2916 & 643.5 & PD \\
\hline 16 & 24646 & 2.12 & 3.97 & / & / & 14428 & 1.98 & 3.17 & 717 & 586.4 & SD \\
\hline
\end{tabular}

Table 3: Means \pm standard deviations and ranges of the ${ }^{18}$ F-FET PET/MR-derived parameters at baseline and after 2 cycles of regorafenib. FET ${ }_{\text {vol/pat }}$ and $\mathrm{ADC}_{\mathrm{vol} / \text { pat }}$ are the pathological segmented FET and $\mathrm{ADC}$ volumes, expressed in $\mathrm{mm}^{3}$; $\mathrm{TBR}_{\text {mean }}$ (mean Tumor-to-Background Ratio) and TBR max $_{\text {(maximum }}$ Tumor-to-Background Ratio) were calculated as the mean and maximum uptakes, respectively, of the FET-positive area normalized for the mean background uptake; mean $A D C_{\text {vol/pat }}$ is the mean $A D C$ value in the $A D C_{\text {vol/pat }}$ volume.

\begin{tabular}{|lllll|}
\hline PARAMETER & baseline ${ }^{18}$ F-fet pet/mr & POST-REGORAFENIB ${ }^{18}$ F-FET PET/MR \\
\hline fet $_{\text {vol/pat }}$ & $14161 \pm 15387 \mathrm{~mm}^{3}$ & $1242-57593 \mathrm{~mm}^{3}$ & $25807 \pm 34548 \mathrm{~mm}^{3}$ & $1486-111268 \mathrm{~mm}^{3}$ \\
\hline TBR $_{\text {max }}$ & $3.22 \pm 0.65$ & $2.18-4.29$ & $3.24 \pm 0.70$ & $2.11-4.64$ \\
\hline Tbr $_{\text {mean }}$ & $1.93 \pm 0.14$ & $1.69-2.2$ & $1.99 \pm 0.18$ & $1.69-2.35$ \\
\hline adc $_{\text {vol/pat }}$ & $438 \pm 363 \mathrm{~mm}^{3}$ & $15-1252 \mathrm{~mm}^{3}$ & $847 \pm 887 \mathrm{~mm}^{3}$ & $41-2916 \mathrm{~mm}^{3}$ \\
\hline mean adc $_{\text {vol/pat }}$ & $597 \pm 97 * 10^{-6} \mathrm{~mm}^{2} / \mathrm{s}$ & $383-677 * 10^{-6} \mathrm{~mm}^{2} / \mathrm{s}$ & $624 \pm 67 * 10^{-6} \mathrm{~mm}^{2} / \mathrm{s}$ & $462-734 * 10^{-6} \mathrm{~mm}^{2} / \mathrm{s}$ \\
\hline
\end{tabular}

Table 4: Variations in the ${ }^{18} \mathrm{~F}$-FET PET/MR-derived parameters in the subjects grouped according to RANO response category. $\mathrm{FET}_{\text {vol/pat }}$ and $\mathrm{ADC}$ vol/pat are the pathological segmented FET and ADC volumes; TBR mean is the mean Tumor-to-Background Ratio and TBR max $_{\text {is }}$ the maximum Tumor-to-Background Ratio; mean $A D C_{\text {vol/pat }}$ is the mean $A D C$ value in the $A D C_{\text {vol/pat }}$ volume. RANO criteria were used to sort the patients into response categories: $S D=S$ table Disease; $P D=$ Progressive Disease. 


\begin{tabular}{|c|c|c|c|c|}
\hline \multirow[t]{2}{*}{ PARAMETER } & \multicolumn{2}{|l|}{ SD } & \multicolumn{2}{|l|}{ PD } \\
\hline & mean \pm standard deviation & range & mean \pm standard deviation & range \\
\hline$\Delta$ fet $_{\text {pat } / v o l}$ & $-1160 \pm 5138 \mathrm{~mm}^{3}$ & $-10218-5895 \mathrm{~mm}^{3}$ & $21605 \pm 36769 \mathrm{~mm}^{3}$ & $-18107-88484 \mathrm{~mm}^{3}$ \\
\hline$\Delta$ fet $_{\mathrm{pat} / \mathrm{vol}}(\%)$ & $70 \pm 199 \%$ & $-41-474 \%$ & $168 \pm 261 \%$ & $-76-823 \%$ \\
\hline$\Delta \mathrm{TBR}_{\max }$ & $0.1 \pm 0.76$ & $-0.8-1.34$ & $-0.05 \pm 0.74$ & $-1.31-1$ \\
\hline$\Delta \mathrm{TBR}_{\max }(\%)$ & $5 \pm 29$ & $-21-58$ & $0 \pm 21$ & $-38-27 \%$ \\
\hline$\Delta \mathrm{Tbr}_{\text {mean }}$ & $0.03 \pm 0.14$ & $-0.14-0.28$ & $0.09 \pm 0.18$ & $-0.2-0.41$ \\
\hline$\Delta \mathrm{Tbr}_{\text {mean }}(\%)$ & $1 \pm 8 \%$ & $-6-15 \%$ & $5 \pm 9 \%$ & $-9-21 \%$ \\
\hline$\Delta \mathrm{adc}_{\mathrm{pat} / \mathrm{vol}}$ & $33 \pm 448 \mathrm{~mm}^{3}$ & $-844-425 \mathrm{~mm}^{3}$ & $501 \pm 1200 \mathrm{~mm}^{3}$ & $-1074-2682 \mathrm{~mm}^{3}$ \\
\hline$\Delta \mathrm{adc}_{\mathrm{pat} / \mathrm{vol}}(\%)$ & $297 \pm 645 \%$ & $-92-1606 \%$ & $554 \pm 940 \%$ & $-94-2460 \%$ \\
\hline$\Delta$ mean adc $c_{\text {pat/vol }}$ & $21 \pm 85 * 10^{-6} \mathrm{~mm}^{2} / \mathrm{s}$ & $-67-182 * 10^{-6} \mathrm{~mm}^{2} / \mathrm{s}$ & $35 \pm 34 * 10^{-6} \mathrm{~mm}^{2} / \mathrm{s}$ & $-20-88 * 10^{-6} \mathrm{~mm}^{2} / \mathrm{s}$ \\
\hline$\Delta$ mean adc $\mathrm{pat} / \mathrm{vol} \%$ & $6 \pm 21 \%$ & $-11-48 \%$ & $6 \pm 6 \%$ & $-3-15 \%$ \\
\hline
\end{tabular}

\section{Figures}
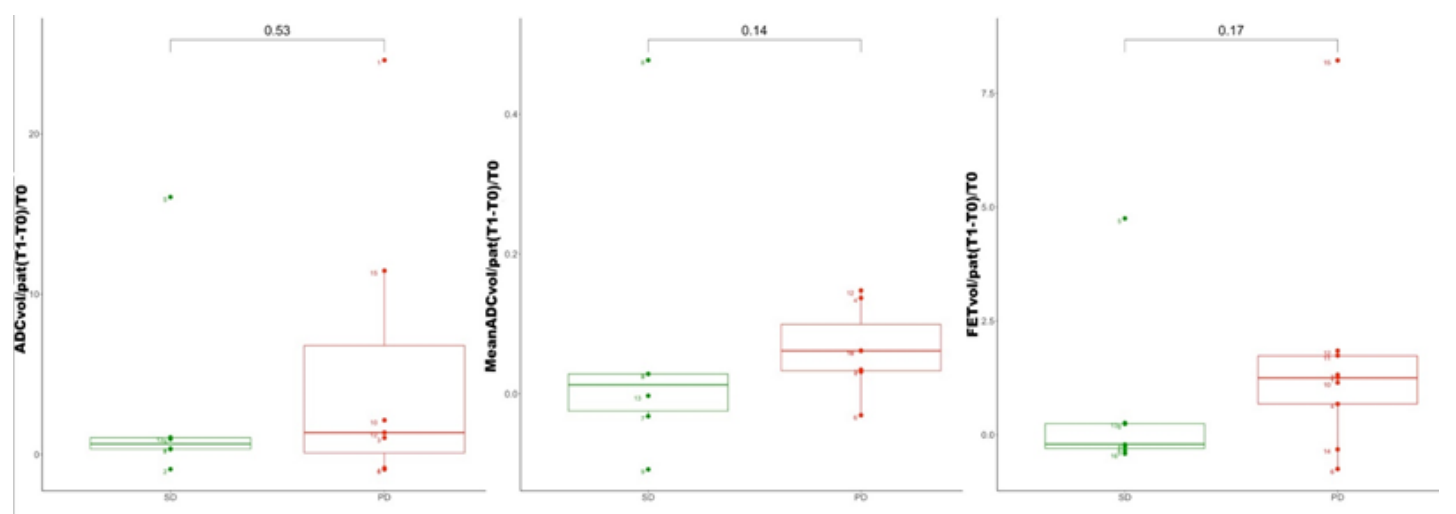

\section{Figure 1}

Variations in the ADC-derived parameters after regorafenib in study patients sorted into response groups according to RANO criteria. Left, the variation in

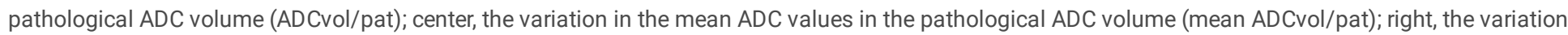
in pathological FET volume (FETvol/pat). SD = Stable Disease; $\mathrm{PD}=$ Progressive Disease 


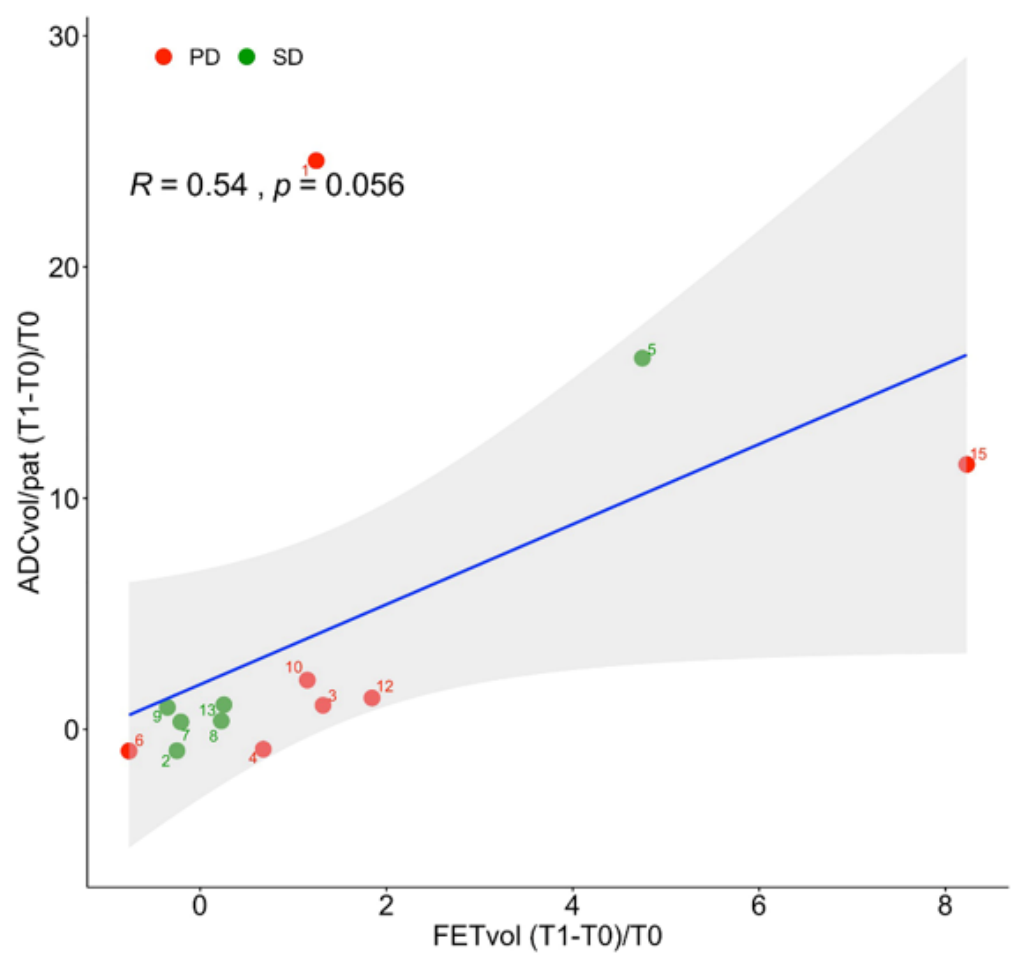

Figure 2

Scatter plots of the variation in pathological ADC volume (ADCvol/pat) versus the corresponding pathological FET volume (FETvol/pat) after 2 cycles of treatment with regorafenib. Red and green dots represent the patients presenting with progressive and stable disease after regorafenib, respectively. The regression line is shown in blue, and the gray area represents the $95 \%$ confidence interval. SD = Stable Disease; PD = Progressive Disease.

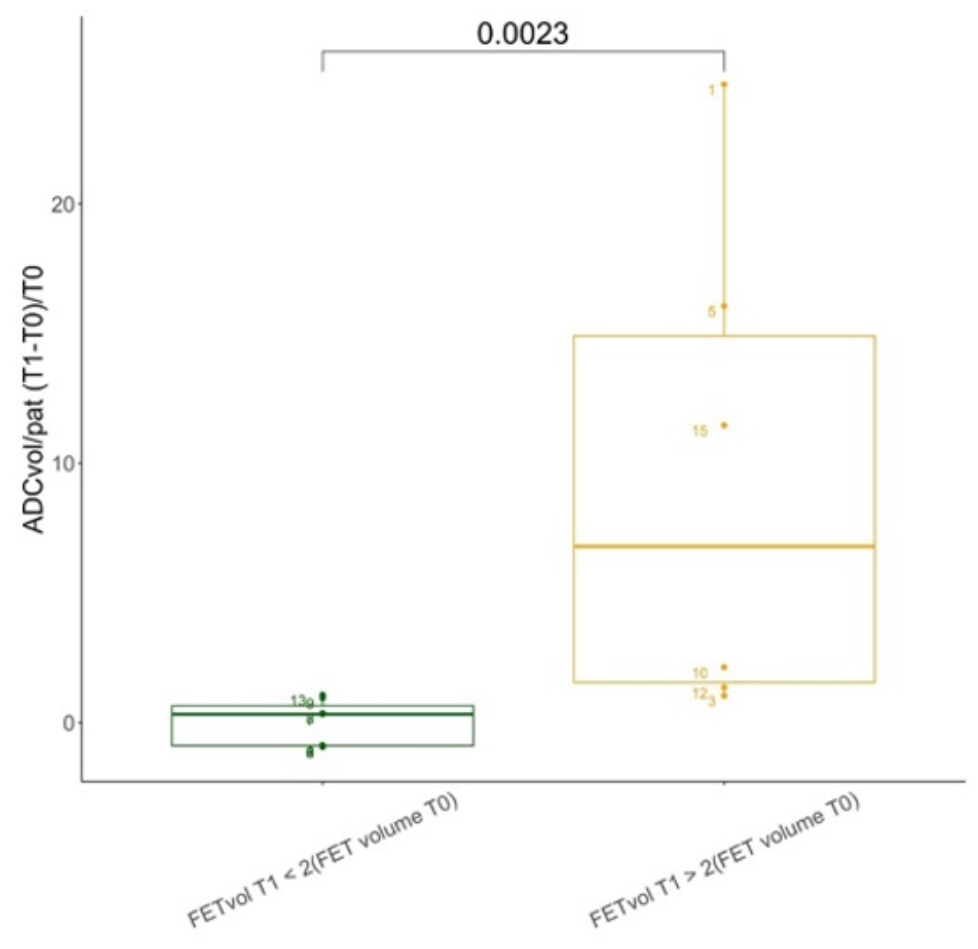

Figure 3

Box plots of the variation in the pathological ADC volume (ADCvol/pat) after two cycles of regorafenib. Study patients were subdivided into two groups: one including patients with at least a duplication in FETvol/pat after treatment with regorafenib (in yellow), the other including subjects with a less than twofold increase in FETvol/pat (in green). 


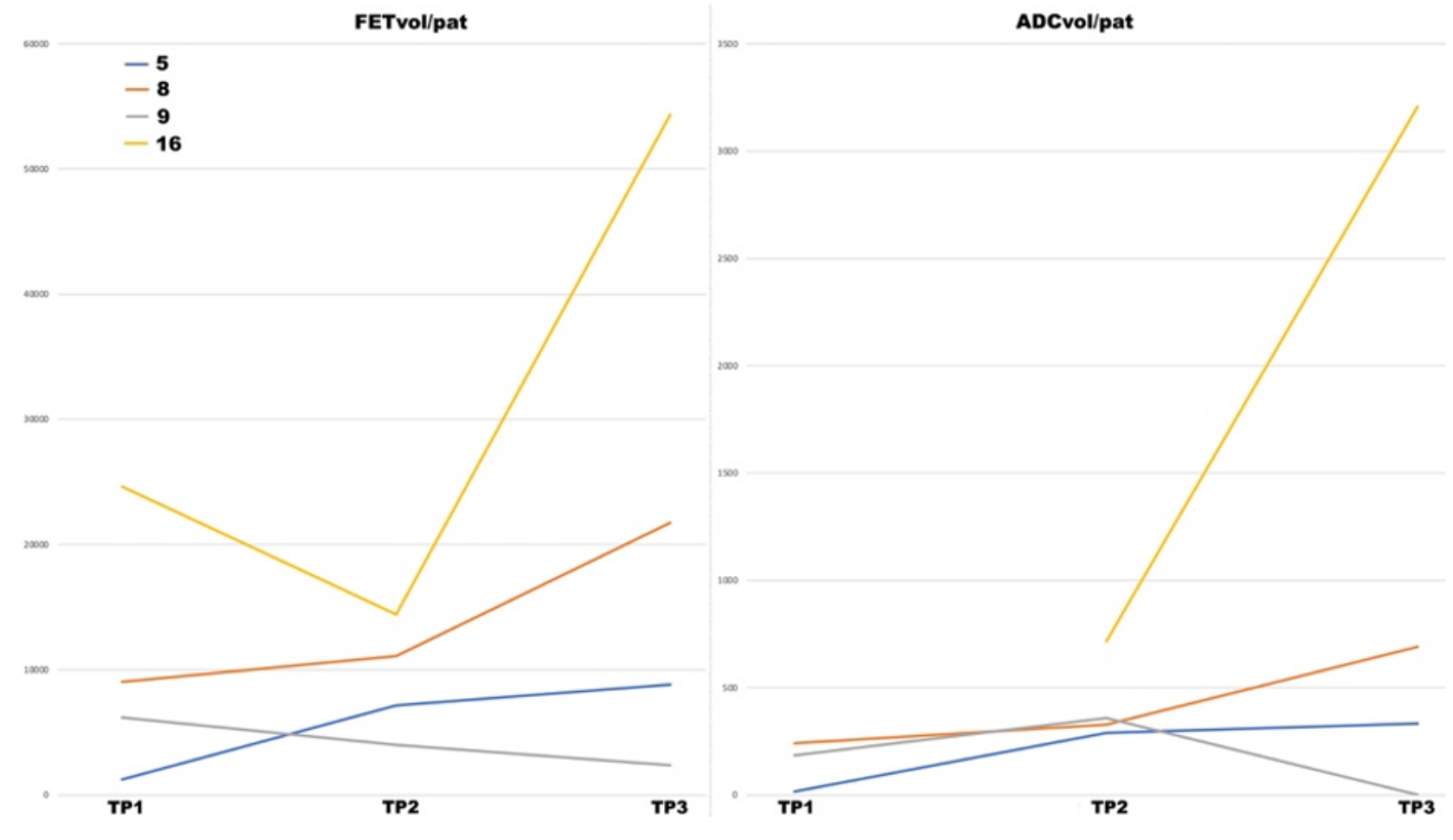

\section{Figure 4}

Variations in the pathological FET volume (FETvol/pat) and pathological ADC volume (ADCvol/pat) between timepoints (TP1, TP2 and TP3 representing, respectively, the first baseline PET/MR before regorafenib, the second PET/MR after two cycles of regorafenib, and the third PET/MR after six cycles of regorafenib). Subjects 5, 8, 9 and 16 were classified (at the latest PET/MR) as progressive disease $(8,16)$ or stable disease $(5,9)$ according to RANO criteria.

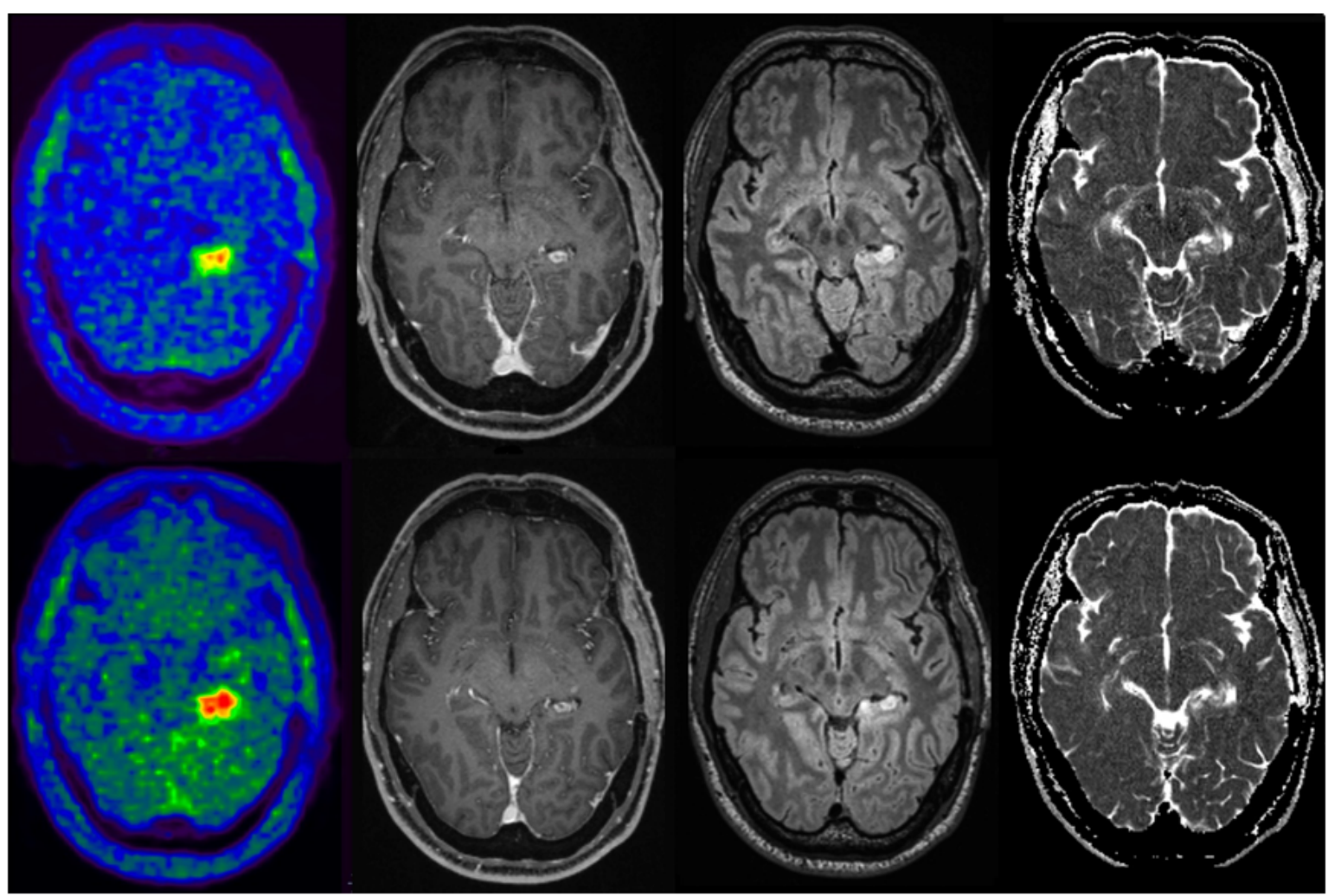

Figure 5 
Axial 18F-FET PET/MR images before (upper row) and after (lower row) treatment with regorafenib in one of the study patients (\#5). 1st column: 18F-FET PET; 2nd column: post-contrast T1-weighted; 3rd column: FLAIR; 4th Column: ADC maps. The increase in 18F-FET uptake after treatment with regorafenib is evident in lower row 1 st column compared with upper row even though no significant increases in the contrast-enhanced and FLAIR altered areas are visible. The analysis revealed an increase in the pathological ADC-volume after regorafenib, even though it was barely detectable qualitatively on the native images.

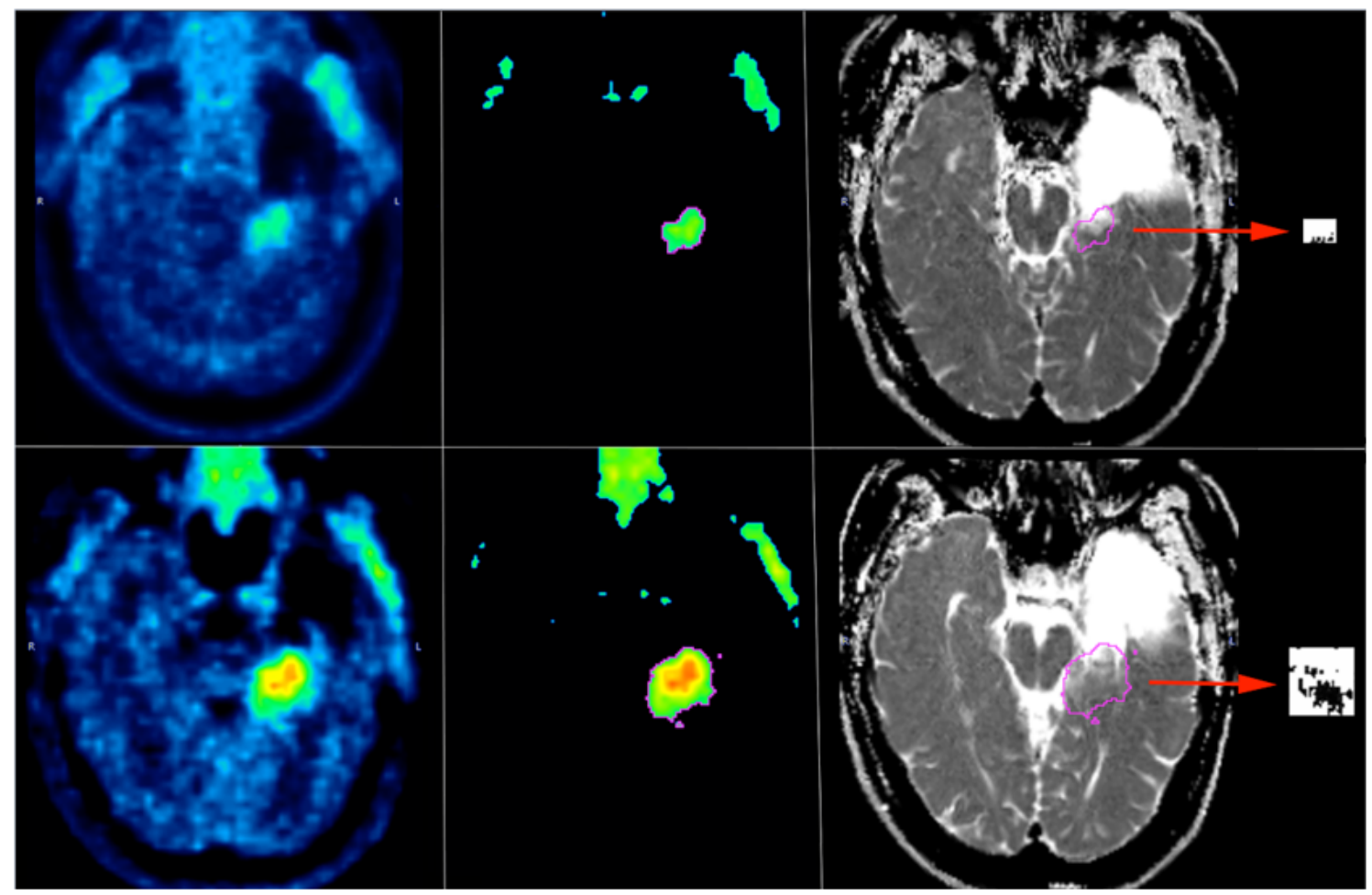

\section{Figure 6}

Overview of the methodology used to segment the pathological 18F-FET and ADC volumes in one of the study patients before (upper row) and after (lower row) treatment with regorafenib. Left: native 18F-FET PET images showing an area of pathological radiotracer uptake in the left mesial temporal lobe; center: the segmented pathological FET volume (FETvol/pat) determined through a 3D semiautomatic contouring process and excluding areas with 18FFET uptake less than 1.6 times the mean background activity; right: the pathological FET volume superimposed onto ADC images and (in boxes pointed by red arrows) the resulting pixels (black dots) with values below the mean ADC background. 